De la Calle Cabrera, A.M., Guzmán-Simón, F. y García-Jiménez, E. (2019). Los precursores cognitivos tempranos̄ de la lectura inicial: un modelo de aprendizaje en niños de 6 a 8 años. Revista de Investigación Educativa, 37(2), 345-361. DOI: http://dx.doi.org/10.6018/rie.37.2.312661

\title{
Los precursores cognitivos tempranos de la lectura inicial: un modelo de aprendizaje en niños de 6 a 8 años
}

\section{Early cognitive precursors of initial reading: a learning model in children aged 6 to 8}

\author{
Ana María de la Calle Cabrera ${ }^{(*)}$, Fernando Guzmán-Simón ${ }^{(* *)}$ y Eduardo García-jiménez ${ }^{(* * *)}$ \\ *Departamento de Comunicación y Educación, Universidad Loyola Andalucía \\ **Departamento de Didáctica de la Lengua y la Literatura y Filologías Integradas, Universidad de Sevilla \\ ***:Departamento de Métodos de Investigación y Diagnóstico en Educación, Universidad de Sevilla
}

\begin{abstract}
Resumen
Los estudios en alfabetización temprana han analizado las relaciones antecedentes entre las habilidades cognitivas en la Educación Infantil y el logro de la lectura durante el proceso de aprendizaje instructivo. Esta investigación describe la capacidad de predicción de ciertas habilidades cognitivas en las primeras etapas instruccionales del aprendizaje lector en español. Los participantes de este estudio fueron 362 niños españoles evaluados por primera vez en $2^{\circ} y$ $3^{\circ}$ de Educación Infantil. Los instrumentos empleados en las evaluaciones de las competencias lingüísticas han sido la Batería de Inicio a la Lectura, la Batería de Evaluación de los Procesos Lectores, The Rapid Automatized Naming Test y el Test de Lectura y Escritura en Español. Los resultados delimitaron un modelo de ecuaciones estructurales para la predicción del rendimiento lector inicial en $1^{\underline{o}}$ y $2^{\underline{o}}$ de Educación Primaria. El modelo alcanzado, Modelo de Aprendizaje en la Lectura Inicial, delimitó las relaciones entre las habilidades del lenguaje oral y las habilidades relacionadas con el código escrito que actuaron como precursores del rendimiento lector inicial. El desempeño en la tarea de velocidad de denominación de letras en Educación Infantil se presentó como el mejor precursor de la eficiencia lectora en $1^{\underline{o}}$ y $2^{\underline{o}}$ de Educación Primaria.
\end{abstract}

Correspondencia: Ana María De la Calle Cabrera, anamaria.decalleca@gmail.com, Avda. República Saharaui s/n, 11519, Puerto Real, Cádiz (España). 
Los hallazgos del estudio realizan una contribución significativa a la investigación en alfabetización temprana en español como referente para el desarrollo de actuaciones pedagógicas y el logro de éxito lector.

Palabras claves: proceso de aprendizaje; capacidad cognitiva; lectura; evaluación predictiva.

\section{Abstract}

Early literacy studies analyzes the antecedent relationships between cognitive skills in preschool education and achievement of reading during the instructional learning process. This study describes the predictive capacity of certain cognitive abilities in the early instructional stages of Spanish learning. The participants of this study was 362 Spanish children evaluated in 2nd and 3rd year of Early Childhood Education (children aged 4 to 6 years) at the first time. The instruments used in the assessments of language skills have been the Beginning Reading Battery, the Reading Process Assessment Battery, The Rapid Automatized Naming Test and the Reading and Writing Test in Spanish. The results delimited a model of structural equations for the prediction of initial reading performance in 1st and 2nd year of Primary Education (6 to 8 years). The model reached, Learning Model in the Initial Reading, delimited the relations between the oral language skills and the skills related to the code that acts as precursors of the initial reader performance. The performance in the task of letters-naming speed at 2 nd and 3rd of Early Childhood Education was presented as the best precursor of reading efficiency at 1st and 2nd year of Primary Education. The findings of study make a significant contribution to early literacy research in Spanish as a reference to develop of pedagogical actions for the achievement of reader success.

Palabras claves: learning process; cognitive ability; reading; predictive evaluation.

\section{Introducción}

La alfabetización es objeto de estudio en los distintos niveles educativos, desde la educación infantil hasta los niveles superiores o edad adulta (Frost, Madsbjerg, Niedersoe, Olofsson \& Sorensen, 2005; Mol \& Bus, 2011). Las habilidades cognitivas que poseen las personas condicionan el aprendizaje lector en los distintos niveles. Por ejemplo, el estudio de Adlof, Catts \& Lee (2010) demostró con 433 niños ${ }^{1}$ estadounidenses que los precursores del rendimiento lector fueron sensibles al paso del tiempo. La conciencia fonológica, la gramática, la inteligencia, la memoria de trabajo, la comprensión oral y el vocabulario expresivo obtuvieron valor predictivo para el rendimiento lector en $8^{\circ}$. Sin embargo, el vocabulario comprensivo, la velocidad de denominación y el conocimiento de las letras sólo fueron precursores del rendimiento lector en $2^{\underline{o}}$ de Educación Primaria.

Las habilidades cognitivas desempeñan un rol predictivo diferenciado en el proceso de aprendizaje de la lectura. Por esta razón, la investigación sobre los precursores de la lectura analiza el valor predictivo de las habilidades cognitivas concretando las etapas

1 Los autores hacen uso en este artículo del masculino como género no marcado. Los términos "niños", "alumnos", "padres" y "profesores" designan ambos sexos. 
del proceso de aprendizaje lector (Manis, Doi \& Badha, 2000; Oakhill \& Cain, 2012). En particular, los estudios sobre alfabetización temprana han analizado las relaciones antecedentes entre las habilidades cognitivas en la educación infantil y el logro de la lectura durante el proceso de aprendizaje instructivo (Kendeou, Van-den-Brock, White \& Lynch, 2009; Torppa, Lyytinen, Erskine, Eklund \& Lyytinen, 2010; Lonigan, Schatschneider, Westberg \& NELP, 2008).

\section{Los precursores cognitivos tempranos de la lectura}

Los precursores cognitivos tempranos de la lectura mejor documentados en los primeros niveles de escolarización han sido las habilidades del lenguaje oral y tres habilidades relacionadas con el código: la conciencia fonológica, la velocidad de denominación y el conocimiento de las letras (Kirby, Desrochers, Roth \& Desrochers, 2008; Lonigan, 2006). Sin embargo, la investigación ha encontrado particularidades en el valor predictivo de estas habilidades cognitivas de acuerdo con el grado de transparencia lingüística.

Un referente de estos hallazgos es el estudio de Georgiou, Parrila y Papadopoulos (2008), que encontró diferencias en el valor predictivo de la conciencia fonológica y de la velocidad de denominación entre 110 niños de habla inglesa y 70 niños griegos en $1^{1}$ y $2^{\circ}$ curso (entre los 6 y 8 años). La conciencia fonológica fue un precursor mejor para las habilidades de decodificación en los niños de habla inglesa $\left(\mathrm{R}_{\mathrm{CF}-\mathrm{HD}}^{2}=.42\right)$ que para los niños de habla griega $\left(R_{\text {CF-HD }}^{2}=.35\right)$ en $1^{\circ}$ (6-7 años). En cambio, la velocidad de denominación sólo actúo como precursor de las mismas destrezas en 1ํㅜ (6-7 años) para los niños griegos $\left(\mathrm{R}_{\mathrm{VD}-\mathrm{HD}}^{2}=(-.31)\right)$. La conciencia fonológica y la velocidad de denominación explicaron porcentajes similares de la varianza de la eficiencia lectora de $1^{\circ}$ (6-7 años) en los niños de habla inglesa $\left(R_{C F-E L}^{2}=.19 ; R_{\mathrm{VD}-\mathrm{EL}}^{2}=(-.20)\right)$, mientras la velocidad de denominación predijo el $56 \%$ de la varianza en los niños griegos. Sin embargo, las destrezas lectoras de 1ํㅡ (6-7 años), en la conciencia fonológica y la velocidad de denominación para ambos grupos, explicaron la misma varianza única del rendimiento lector de 20 (7-8 años) (entorno al 70\% de la varianza).

En definitiva, el grado de transparencia de las lenguas no fue el único factor que intercede en las conclusiones de los estudios sobre los precursores cognitivos de la lectura. Los hallazgos de estos estudios fueron alcanzados de acuerdo a ciertas singularidades en el diseño metodológico, como las decisiones en la elección de las variables, la temporalización de las evaluaciones, el procedimiento y los materiales empleados para tomar las medidas. No obstante, estas investigaciones alcanzan conclusiones análogas, pese a las características lingüísticas de la muestra y las metodologías aplicadas a la investigación (Dickinson, McCabe, Anastasopoulos, Peisner-Feinberg \& Poe, 2003; Paris, 2005).

\section{Las habilidades del lenguaje oral}

Las habilidades del lenguaje oral sustentan el proceso de adquisición de la lectura (Kendeou et al. 2009; Silvén, Poskiparta, Niemi \& Vorten, 2007; Storch \& Whitehurst, 2002). Estas habilidades realizan una contribución directa al rendimiento lector. Los 
hallazgos de Whitehurst (1996) mostraron que el rendimiento lector desde los 6 a los 8 años era determinado por el conocimiento de letras, la sensibilidad fonológica y la escritura emergente $\left(.49<\mathrm{R}^{2}<.60\right) \mathrm{y}$, en menor medida, por el nivel de vocabulario comprensivo y expresivo que poseen los niños a los $4-5$ años $\left(R^{2}=.17\right)$ (Whitehurst \& Lonigan, 1998). En el mismo sentido, el estudio de Kendeou et al. (2009) demostró en 133 niños estadounidenses que las habilidades del lenguaje oral (vocabulario y comprensión oral y audiovisual) explicaban las habilidades de descodificación a los 4 y 5 años $\left(\mathrm{R}^{2}{ }_{\text {LO-HD }}=.53\right)$. Mientras, el trabajo de Catts, Herrera, Nielsen y Bridges (2015) subrayó que las habilidades de lenguaje oral (vocabulario y gramática) a estas edades predicen las habilidades de decodificación posterior (en $2^{0}$ curso) en una muestra similar $\left(\mathrm{R}_{\text {LO-HD }}^{2}=.32\right)$. Esta investigación resaltó la existencia de correlaciones entre las habilidades del lenguaje oral y las habilidades relacionadas con el código con valores de r entre.40 y.70 ( $\left.\mathrm{r}_{\mathrm{CL}-\mathrm{LO}}=.46 ; \mathrm{r}_{\mathrm{CF}-\mathrm{LO}}=.67 ; \mathrm{r}_{\mathrm{VD}-\mathrm{LO}}=.37\right)$.

Las habilidades del lenguaje oral favorecen también el desarrollo de otras habilidades cognitivas relacionadas con el código a edades tempranas. Schatschneider, Fletcher, Francis, Carlson y Foorman (2004) realizaron un estudio con niños estadounidenses desde Kinder (5-6 años) a $2^{\underline{o}}$ (7-8 años) en el que analizaron el valor predictivo de la conciencia fonológica, conocimiento de las letras, la velocidad de denominación, el vocabulario, la capacidad perceptiva y el lenguaje oral (morfosintaxis). Sus hallazgos demostraron correlaciones entre la conciencia fonológica a los 5 y 6 años y el conocimiento morfonsintáctico expresivo y comprensivo de $1^{\circ}(6-7$ años $)\left(\mathrm{r}_{\mathrm{CF}-\mathrm{CME}}=.46\right.$; $\mathrm{r}_{\mathrm{CF}-\mathrm{CMC}}$ $=.39)$ y de $2^{o}$ (7-8 años) $\left(\mathrm{r}_{\mathrm{CF}-\mathrm{CME}}=.42 ; \mathrm{r}_{\mathrm{CF}-\mathrm{CMC}}=.31\right)$.

\section{Las habilidades relacionadas con el código: la conciencia fonológica, el conoci- miento de las letras y la velocidad de denominación}

Los estudios sobre precursores tempranos de la lectura inicial coinciden en que el valor predictivo mayor reside en, al menos, una de estas tres habilidades cognitivas. Así, algunos estudios han destacado la conciencia fonológica como el mejor precursor. Evans, Bell, Shaw, Moretti y Page (2006) concluyeron que la conciencia fonológica explicaba el 24\% de la varianza de las habilidades de decodificación en 1ำ (6-7 años) (en niños canadienses de lengua inglesa). Mientras, los hallazgos de Silvén et al. (2007) apoyaron que la conciencia fonológica a los 5 años determinó la lectura de palabras a los 8 años $\left(\mathrm{R}_{\text {CF-LP }}^{2}=.41\right)$ en una muestra de 56 niños finlandeses. En el mismo sentido, Suárez-Coalla, García-De-Castro y Cuetos (2013) determinaron con una muestra española que el nivel de conciencia fonémica que poseen los prelectores es el mejor predictor de la exactitud lectora 18 meses después.

Numerosas investigaciones han encontrado mayor valor predictivo en el conocimiento de las letras o en la velocidad de denominación. Muter, Hulme, Snowling y Stevenson (2004) evidenciaron que la conciencia fonológica a los 4 y 5 años determinó un $21 \%$ de varianza de las habilidades de decodificación un año más tarde, mientras que el conocimiento de las letras explicó el 63\% de su varianza (en 90 sujetos ingleses). Asimismo, la investigación de Torppa et al. (2010) descubrió con 198 niños finlandeses que el conocimiento de las letras era la variable con mayor peso predictivo a los 5 y 5.5 años para la lectura de palabras y pseudopalabras en $2^{\circ}\left(8-9\right.$ años) $\left(R^{2}=.34\right)$. Sin embargo, 
otros estudios han encontrado el mayor valor predictivo en la velocidad en que las letras son denominadas. A título de ejemplo, la investigación de González-Valenzuela, Díaz-Giráldez y López-Montiel (2016) detectó en una muestra de 116 niños españoles que la conciencia fonémica, la memoria fonológica y la velocidad de denominación alfanumérica a los 6 años fueron los predictores de la precisión de lectura para palabras y pseudopalabras. Mientras, la velocidad de denominación alfanumérica tuvo una contribución única a la velocidad y eficiencia lectora $\left(\mathrm{R}^{2}<.30\right.$ en ambos casos). De este modo, la velocidad de denominación de letras indicaría indirectamente el conocimiento de las letras no consolidadas (Neuhaus, Foorman, Francis \& Carlson, 2001).

Otros estudios demostraron que la combinación temprana de dos de estas habilidades cognitivas, la conciencia fonológica, el conocimiento de las letras y la velocidad de denominación, mejoraron el valor predictivo de la lectura en $1^{\circ}$ y $2^{o}$ (6-8 años). Como muestra Storch y Whitehurst (2002), la conciencia fonológica y el principio de impresión (nombre de las letras, identificación letra-sonido e identificación de signos lingüísticos) determinó en gran medida el rendimiento lector en 1ำ (6-7 años) $\left(R^{2}=.76\right)$ y $2^{\circ}$ (7-8 años) $\left(R^{2}=.55\right)$. Asimismo, Kendeou et al. (2009) hallaron que la conciencia fonológica, el reconocimiento de letras y las habilidades de descodificación en 1ำ (6-7 años) explicaban la identificación de palabras en $2^{2}$ grado (7-8 años) $\left(R^{2}=.76\right)$; y las habilidades de decodificación en $2^{\circ}$, la comprensión lectora $\left(\mathrm{R}_{\mathrm{HD}-\mathrm{CL}}^{2}=.29\right)$. Mientras, los hallazgos de Ferroni, Diuk y Mena (2016) mostraron el valor predictivo, a principios de $1^{\underline{0}}$, del conocimiento de las letras para la lectura de palabras al final de $1^{\mathrm{o}}\left(.31<\mathrm{R}^{2}<.38\right)$ y de la velocidad de denominación para la lectura de palabras de $2^{\circ}\left(R^{2}=.19\right)$ en una muestra de niños argentinos.

Las tres habilidades cognitivas, en su conjunto, han actuado como el mejor precursor temprano de la lectura inicial. Por citar un caso, los resultados en el estudio de Schatschneider et al. (2004) mostraron que la conciencia fonológica junto con las medidas de nombre de la letra y del conocimiento sonido de la letra y la velocidad de nombrado fueron buenos predictores de múltiples resultados de lectura de los grados $1^{\circ}$ y $2^{\circ}$ (niños estadounidenses). La conciencia fonológica, el conocimiento del nombre de las letras y la velocidad de denominación de letras al inicio de Kinder (5-6 años) obtuvieron el mayor valor predictivo tanto para la comprensión de oraciones a finales de $1^{\mathrm{o}}\left(6-7\right.$ años) $\left(\mathrm{R}^{2}=.30\right)$ y $2^{\mathrm{o}}\left(7-8\right.$ años) $\left(\mathrm{R}^{2}=.30\right)$ como para la decodificación de $1^{\circ}\left(\mathrm{R}^{2}=.37\right)$ y $2^{\circ}\left(\mathrm{R}^{2}=.32\right)$. Estas tres habilidades cognitivas, la conciencia fonológica, el conocimiento del nombre y sonido de las letras y la velocidad de denominación de letras, obtuvieron el mayor valor predictivo también para la eficiencia lectora en $1^{\mathrm{o}}$

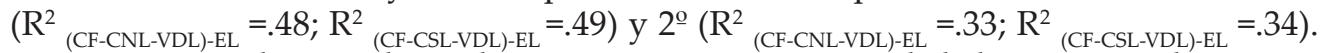

En resumen, los estudios sobre precursores tempranos de la lectura inicial permiten deducir que la conciencia fonológica, el conocimiento de las letras y la velocidad de denominación fueron los mejores precursores tempranos de la lectura (véase tabla 1).

Tabla 1

Sintesis sobre los precursores tempranos de la lectura al inicio de la instrucción formal 


\begin{tabular}{|c|c|c|c|c|c|c|c|}
\hline & & \multirow{2}{*}{ LO } & \multicolumn{3}{|c|}{$\begin{array}{l}\text { Habilidades relacionadas } \\
\text { con el código }\end{array}$} & \multicolumn{2}{|c|}{$\begin{array}{c}\text { Rendimiento } \\
\text { Lector }\end{array}$} \\
\hline & & & $\mathrm{CF}$ & VD & CL & DL & CLec \\
\hline $\begin{array}{l}\text { Whitehurst (1996) (Whitehurst } \\
\text { Lonigan, 1998) }\end{array}$ & $\&$ & S & $S\left({ }^{*}\right)(* *)$ & $N\left({ }^{*}\right)(* *)$ & $S\left({ }^{*}\right)(* *)$ & $S$ & $S$ \\
\hline Storch y Whitehurst (2002) & & S & $S(*)(* *)$ & $\mathrm{N}$ & $\mathrm{N}$ & $S$ & S \\
\hline Muter, et al. (2004) & & S & S & $\mathrm{N}$ & $S(*)(* *)$ & S & $S$ \\
\hline Schatschneider et al. (2004) & & S & $S(*)(* *)$ & $S(*)(* *)$ & $S(*)(* *)$ & S & $S$ \\
\hline Evans et al. (2006) & & S & S & S & S & $\mathrm{N}$ & $\mathrm{N}$ \\
\hline Silvén et al. (2007). & & S & $S(*)$ & $\mathrm{N}$ & $\mathrm{N}$ & S & $\mathrm{N}$ \\
\hline Torppa et al. (2010) & & S & S & $S(* *)$ & $\mathrm{N}$ & S & S \\
\hline Suárez-Coalla et al. (2013) & & $\mathrm{N}$ & $S(*)$ & $S(*)$ & $\mathrm{N}$ & S & $\mathrm{N}$ \\
\hline Catts et al. (2015) & & S & $S\left({ }^{* *}\right)$ & S & $S(* *)$ & $S$ & S \\
\hline González-Valenzuela et al. (2016) & & $\mathrm{N}$ & $S(*)$ & $S(*)$ & $\mathrm{N}$ & S & $\mathrm{N}$ \\
\hline Ferroni et al. (2016) & & $\mathrm{N}$ & S & $S\left({ }^{* *}\right)$ & $S(*)$ & S & $\mathrm{N}$ \\
\hline
\end{tabular}

Nota: $\mathrm{LO}=$ Habilidades del lenguaje oral; $\mathrm{CF}=$ conciencia fonológica; $\mathrm{VD}=$ velocidad de denominación; $\mathrm{CL}=$ conocimiento de las letras; $\mathrm{DL}=$ decodificación lectora; $\mathrm{CLec}=$ Comprensión lectora; $\mathrm{S}=$ Sí fue evaluada; $\mathrm{N}=$ No fue evaluada; $\left({ }^{*}\right)=$ mejor precursor temprano del rendimiento lector en $1^{\mathrm{o}} ;\left(^{* *}\right)=$ mejor precursor temprano del rendimiento lector en $2^{\circ}$.

\section{Método}

\section{Objetivos}

El estudio tuvo por propósito averiguar cuáles son las habilidades cognitivas que actúan como precursores de la lectura en españolen la primera etapa de su aprendizaje instruccional formal. La delimitación de los precursores de la lectura en el primer y segundo curso resulta fundamental, ya que estos asientan las bases del aprendizaje instruccional de la lectura. Con este fin, se examinó las habilidades del lenguaje oral y las habilidades relacionadas con el código en niños españoles de 4 a 6 años de edad, y el rendimiento lector dos años más tarde. Los objetivos marcados en este estudio fueron los siguientes:

1. Determinar un modelo de predicción en el que se incluyan todas las habilidades cognitivas que actúen como precursores tempranos de la lectura al inicio de su aprendizaje instruccional formal.

2. Describir el rol predictivo de las habilidades del lenguaje oral en el modelo de predicción.

3. Analizar el valor predictivo de la conciencia fonológica, el conocimiento de las letras y la velocidad de denominación en el modelo de predicción. 


\section{Población y Muestra}

Este estudio se llevó a cabo en tres colegios públicos españoles inmersos en un entorno socioeconómico medio de la provincia de Cádiz. Los participantes de este estudio fueron 362 niños de 4 a 6 años (edad media $=5.2 ; 47.2 \%$ niñas), 180 alumnos de $2^{\mathrm{o}}$ Educación Infantil (edad media $=4.7 ; 45.6 \%$ niñas) y de 182 alumnos de $3^{\mathrm{o}}$ de Educación Infantil (edad media $=5.7 ; 48.9 \%$ niñas). La selección de los alumnos estuvo condicionada al consentimiento informado de sus progenitores y a la ausencia de necesidades educativas. Este trabajo de investigación siguió la regulación interna para la investigación en Ciencias Sociales requerida por los Comités Éticos de la Universidad de Cádiz y de Sevilla.

\section{Instrumento}

1. Batería de Inicio a la Lectura (BIL 3-6) (Sellés, Martinez \& Vidal-Abarca, 2010). Esta prueba permite medir habilidades cognitivas tempranas con valor predictivo para el éxito lector. La tabla 2 muestra las 13 subpruebas empleadas en este estudio (de las 15 subpruebas de la batería). Sellés et al. (2010) validaron BIL3-6 mediante validez de contenido con el Test de Análisis de la Lectoescritura (TALE) y correlacional. Los coeficientes de alfa de Cronbach hallados por Sellés et al. (2010) en cada subprueba y los calculados en este estudio pueden compararse en la tabla 2.

Tabla 2

Subpruebas empleadas de la Batería de Inicio a la Lectura BIL 3-6

\begin{tabular}{|c|c|c|c|c|}
\hline Subpruebas & Ítems & Descripción de lo evaluado & $\begin{array}{l}\alpha \text { de Cronbach } \\
\text { Sellés et al (2010) }\end{array}$ & $\begin{array}{l}\alpha \text { de Cronbach } \\
\text { en este estudio }\end{array}$ \\
\hline Vocabulario & 8 & $\begin{array}{l}\text { Nivel de vocabulario expresi- } \\
\text { vo mediante la denominación } \\
\text { de imágenes. }\end{array}$ & .69 & .70 \\
\hline Articulación & 15 & $\begin{array}{l}\text { Capacidad de identificar los } \\
\text { sonidos del habla y repetir pa- } \\
\text { labras. }\end{array}$ & .92 & .74 \\
\hline Memoria secuencial auditiva & 8 & $\begin{array}{l}\text { Mide la memoria verbal a cor- } \\
\text { to plazo con una tarea de repe- } \\
\text { tir una secuencia de palabras y } \\
\text { frases. }\end{array}$ & .88 & .75 \\
\hline Percepción visual & 9 & $\begin{array}{l}\text { Capacidad de discriminar en- } \\
\text { tre símbolos y grafías seme- } \\
\text { jantes visualmente. }\end{array}$ & .87 & .73 \\
\hline Reconocimiento de palabras & 10 & $\begin{array}{l}\text { Reconocimiento visual de una } \\
\text { palabra entre no palabras que } \\
\text { integran números y otros sím- } \\
\text { bolos. }\end{array}$ & .77 & .73 \\
\hline Contar palabras & 6 & $\begin{array}{l}\text { Capacidad de segmentar ora- } \\
\text { ciones en palabras. }\end{array}$ & .64 & .73 \\
\hline
\end{tabular}




\begin{tabular}{lcllc}
\hline Subpruebas & Ítems & Descripción de lo evaluado & $\begin{array}{l}\alpha \text { de Cronbach } \\
\text { Sellés et al (2010) }\end{array}$ & $\begin{array}{c}\alpha \text { de Cronbach } \\
\text { en este estudio }\end{array}$ \\
\hline Reconocer Frases & 5 & $\begin{array}{l}\text { Discriminar visualmente si } \\
\text { lo presentado es o no una } \\
\text { oración. }\end{array}$ & .69 & .79 \\
Aislar sílabas y fonemas & 8 & $\begin{array}{l}\text { Identificación de sílabas y } \\
\text { fonemas al principio de pa- } \\
\text { labra. }\end{array}$ & .82 & .73 \\
Funciones de la lectura & 5 & $\begin{array}{l}\text { Funcionalidad del lenguaje } \\
\text { escrito. }\end{array}$ & .72 & .73 \\
$\begin{array}{l}\text { Conocimiento de estructuras } \\
\text { gramaticales }\end{array}$ & 6 & $\begin{array}{l}\text { Identificar si los enunciados } \\
\text { tienen sentido o carecen de } \\
\text { falta de concordancias. } \\
\text { Conceptos básicos }\end{array}$ & 8 & $\begin{array}{l}\text { Conceptos básicos. } \\
\text { Capacidad de segmentar } \\
\text { palabras en sílabas. } \\
\text { Contar sílabas }\end{array}$ \\
Omisión de sílabas & 5 & $\begin{array}{l}\text { Supresión de la primera sílaba } \\
\text { de una palabra. }\end{array}$ & .54 & .69 \\
\hline
\end{tabular}

2. Batería de Evaluación de los Procesos Lectores (PROLEC-R) (Cuetos, Rodríguez, Ruano \& Arribas, 2007). La evaluación mediante esta prueba consistió en la aplicación del subtest de reconocimiento de las letras y de una adaptación del subtest de lectura de pseudopalabras. El subtest de reconocimiento de letras permite medir el conocimiento de 20 de las 27 grafías simples existentes en español (19 consonánticas y 1 vocálica; excepto "a", "e", "i", "o", “ $h$ ", " $\mathrm{k}$ " y “w”), considerándose acierto una respuesta que identifique el nombre, el fonema correspondiente a la letra o el fonema dentro de una estructura fonológica silábica. El subtest de repetición de pseudopalabras evalúa la reproducción de 12 pseudopalabras, 6 cortas y 6 largas, que fueron seleccionadas aleatoriamente del subtest de lectura de pseudopalabras.

Cuetos et al. (2007) validan PROLEC-R mediante una validez concurrente con la prueba Peabody (Dunn, Dunn \& Arribas, 2006). El coeficiente del alfa de Cronbach en el subtest de reconocimiento de letras de.49 es menor al obtenido en este estudio ( $\alpha$ de Cronbach =.76). El coeficiente del alfa de Cronbach en la adaptación del subtest de lectura de pseudopalabras fue de.73.

3. The Rapid Automatized Naming Test (RAN) (Wolf \& Denckla, 2003). El test RAN consiste en realizar 4 subpruebas de nombrado rápido de 50 estímulos en cada una (dígitos, grafías, colores y dibujos) y las puntuaciones se computan en aciertos por segundo. Wolf y Denckla (2003) realizaron una revisión de la prueba de Denckla y Rudel (1976) y emplearon el método de las dos mitades para calcular la fiabilidad de la prueba, obteniendo un valor de.80. En este estudio, el coeficiente del alfa de Cronbach obtenido es de.92.

4. Test de Lectura y Escritura en Español (LEE) (Defior et al., 2006). Este instrumento permite valorar las habilidades de decodificación y la comprensión de textos en términos de eficiencia lectora en $1^{\underline{0}}$ y $2^{\underline{o}}$ de Educación Primaria (6 a 8 años). 
Las subpruebas de eficiencia lectora consisten en la lectura de 40 palabras y 40 pseudopalabras en minúsculas. Los autores hallan un coeficiente de alfa de Cronbach de.80 y.70 para la lectura de palabras en $1^{\mathrm{o}}$ y $2^{\mathrm{o}}$ de Educación Primaria, respectivamente. Los coeficientes de alfa de Cronbach alcanzados en esta investigación fueron superiores a sus medidas en la lectura de pseudopalabras.82 (en $1^{\mathrm{o}}$ ) y.79 (en $2^{\mathrm{o}}$ ). Por otro lado, los índices de fiabilidad en las dos tareas de lectura fueron semejantes en este estudio ( $\alpha$ de Cronbach $=.75$ en lectura de palabras y $\alpha$ de Cronbach $=.72$ en lectura de pseudopalabras).

Por último, las subpruebas de comprensión lectora de textos consisten en la evaluación de la micro y macroestructura de tres textos a partir de preguntas literales e inferenciales y la selección del título y resumen de cada texto. El número de aciertos y el tiempo empleado fueron considerados para el cálculo de la puntuación obtenida en cada subprueba. Los autores hallan un coeficiente de alfa de Cronbach de.78 y.70 para esta subprueba en $1^{\underline{0}}$ y $2^{\underline{o}}$ de Educación Primaria, respectivamente. El índice de fiabilidad es de.78 en este caso. Los autores realizaron validez convergente, discriminante, estudios de confiabilidad de test-retest $(.50<\mathrm{r}<.88)$, y el método de las dos mitades (valores entre.55 y.78).

\section{Procedimiento de recogida y análisis de datos}

Los alumnos fueron evaluados individualmente en dos momentos distintos, puesto que este estudio responde a un enfoque longitudinal prospectivo. El primer momento $\left(\mathrm{t}_{1}\right)$ discurrió en el segundo trimestre del curso escolar de $2^{\mathrm{o}}$ y $3^{\mathrm{o}}$ de Educación Infantil; y el segundo momento $\left(t_{2}\right), 24$ meses después, cuando los alumnos cursaban $1^{\circ}$ y $2^{\circ}$ de Educación Primaria. Los instrumentos fueron seleccionados de acuerdo con las características de los participantes de este estudio (edad y lengua materna). Uno de los investigadores desarrolló las evaluaciones al alumnado individualmente en dos sesiones diferentes (la sesión $\mathrm{t} 1$ y la sesión $\mathrm{t} 2$ ), haciendo uso para ello de un espacio privado que reunía las condiciones ambientales oportunas. La sesión $\mathrm{t} 1$, estuvo dedicada a la evaluación de las capacidades de los niños de los 4 a 6 años en las habilidades del lenguaje oral (vocabulario, conceptos básicos, morfosintaxis, habilidades articulatorias y conocimiento fonológico), el conocimiento de las letras, el conocimiento metalingüístico, la velocidad de denominación y los procesos cognitivos (memoria y percepción); y la sesión $\mathrm{t} 2$, a la evaluación en $1^{\mathrm{o}}$ y $2^{\mathrm{o}}$ de Educación Primaria (6 a 8 años) del rendimiento lector. Así, las habilidades cognitivas precursoras fueron evaluadas mediante tres instrumentos (BIL, RAN, y PROLEC-R) en el $t 1$ y el rendimiento lector, mediante uno (LEE) en el t2. La sesión t1 tuvo una duración aproximada de 35 minutos; mientras, la sesión t 2 fue un poco más breve (25 minutos aproximadamente). Los resultados de las distintas evaluaciones fueron reportadas al equipo directivo, al equipo de orientación educativa y al profesorado, quienes tomaron las medidas educativas pertinentes en alusión al contenido de las mismas.

Los datos fueron analizados mediante los paquetes estadísticos SPSS y AMOS en sus versiones 23. En primer lugar, los datos obtenidos en las distintas pruebas fueron sometidos a análisis multinivel considerando la edad de los niños y el centro de pertenencia como los factores fijos. La tabla 3 de la prueba de efectos inter-sujetos muestra que la presencia de las dos variables no es determinante para los resultados obtenidos en las distintas pruebas (no existen diferencias significativas). En consecuencia, los análisis posteriores consideran los resultados obtenidos en las distintas pruebas por 
la totalidad de los participantes $(n=362)$. Estos consistieron en el análisis de regresión lineal y en la configuración de un modelo de ecuaciones estructurales.

Tabla 3

Efectos inter-sujetos en el análisis multinivel

\begin{tabular}{llccccc}
\hline Origen & Variable dependiente & $\begin{array}{l}\text { Tipo III de suma de } \\
\text { cuadrados }\end{array}$ & gl & $\begin{array}{c}\text { Media } \\
\text { cuadrática }\end{array}$ & F & Sig. \\
\hline Colegio & BIL & 105.431 & 2 & 52.715 & .226 & .798 \\
$*$ * curso & PROLEC & 72.689 & 2 & 36.344 & 1.062 & .347 \\
& RAN & .520 & 2 & .260 & .375 & .688 \\
& LEE & 3.081 & 2 & 1.540 & 2.754 & .065 \\
\hline
\end{tabular}

\section{Resultados}

Las habilidades cognitivas tempranas con valor predictivo para el rendimiento lector en las primeras etapas del aprendizaje instruccional de la lectura se analizaron mediante regresión. Las relaciones de regresión lineal entre las variables predictoras en $2^{\underline{0}}$ y $3^{\circ}$ de Educación Infantil (4 a 6 años) y el rendimiento lector en $1^{\circ}$ y $2^{\circ}$ de Educación Primaria (6 a 8 años) fueron analizadas en primer lugar. La tabla 4 ilustra los precursores tempranos de la lectura inicial. La velocidad de denominación de números, la velocidad de denominación de letras, la capacidad perceptiva y el vocabulario predicen el $41 \%$ de la varianza de la eficiencia en lectura de palabras y el 34\% de la varianza de la eficiencia en lectura de pseudopalabras $\left(\mathrm{R}_{\text {corregido }}^{2}\right.$ ). Además, las tres primeras (la velocidad de denominación de números, la velocidad de denominación de letras, la capacidad perceptiva) junto al conocimiento fonológico (tarea de aislar sílabas y fonemas y de memoria secuencial auditiva) predijeron la eficiencia en comprensión lectora de textos con un $\mathrm{R}_{\text {corregido }}^{2}$ de.46.

Tabla 4

Modelos de regresión lineal entre las variables predictoras y el rendimiento lector

\begin{tabular}{lccccc}
\hline Modelo & $\mathrm{r}$ & $\mathrm{R}^{2}$ & $\mathrm{R}_{\text {corregido }}^{2}$ & $\begin{array}{c}\text { Error típ. de } \\
\text { la estimación }\end{array}$ & $\begin{array}{c}\text { Durbin- } \\
\text { Watson }\end{array}$ \\
\hline $\begin{array}{l}\text { Eficiencia en lectura de palabras } \\
\begin{array}{l}\text { Eficiencia en lectura de pseudo- } \\
\text { palabras }\end{array}\end{array}$ & $.647 \mathrm{a}$ & .419 & .412 & .39321 & 1.872 \\
$\begin{array}{l}\text { Comprensión lectora } \\
\text { Com }\end{array}$ & .343 & .335 & .28455 & 1.939 \\
\hline
\end{tabular}

Nota: Predictores:

a-b. (constante), velocidad de denominación de números, velocidad de denominación de letras, capacidad perceptiva, vocabulario

c. (constante), velocidad de denominación de números, velocidad de denominación de letras, capacidad perceptiva, aislar sílabas y fonemas, memoria secuencial auditiva 
Estos análisis de regresión lineal fueron completados con otros análisis sucesivos que permitieron la delimitación de un modelo de ecuaciones estructurales. El modelo alcanzado recogió el conjunto de los precursores tempranos de la lectura en español de los 6 a los 8 años. Los índices de bondad de ajuste del modelo mostraron índices aceptables $\left(\chi^{2}=101.444 ; \mathrm{df}=68 ; p=.005 ; \mathrm{CFI}=.986\right.$; RMSEA =.037) (véase tabla 5).

\section{Tabla 5}

Indicadores de ajuste del modelo ALICIA

\begin{tabular}{llllllll}
\hline Chi-square & df & Chi-square/df & NFI & RFI & IFI & TLI & CFI \\
\hline 101.444 & 68 & 1.492 & .960 & .938 & .986 & .979 & .986 \\
\hline
\end{tabular}

El análisis de los precursores de la lectura inicial ha permitido el diseño de un modelo de predicción denominado "Modelo de Aprendizaje en la Lectura Inicial" (en adelante. modelo ALICIA). En este modelo, las habilidades del lenguaje oral fueron el punto de partida del aprendizaje lector. Estas habilidades del lenguaje oral (conocimiento semántico y conocimiento morfosintáctico) correlacionaron con las habilidades de discriminación auditiva y de repetición fono-articulatoria (habilidades articulatorias) $\left(\mathrm{R}_{\text {LO-HA }}^{2}=.65\right)$ y con habilidades para manipular los segmentos del habla (conciencia fonológica) $\left(\mathrm{R}_{\mathrm{LO}-\mathrm{CF}}^{2}=.96\right.$.). Asimismo, las habilidades articulatorias correlacionaron a su vez con la conciencia fonológica $\left(\mathrm{R}_{\mathrm{HA}-\mathrm{CF}}^{2}=.72\right)$.

Las habilidades articulatorias y la conciencia fonológica determinaron la velocidad de denominación de letras $\left(\mathrm{R}_{\mathrm{HA}-\mathrm{CF}}^{2}=.43 ; \mathrm{R}^{2}{ }_{\mathrm{CF}-\mathrm{VDL}}=.69\right)$. Esta adquirió una apariencia de multicomponente como una variable mediadora entre la conciencia fonológica y la eficiencia lectora. Las habilidades articulatorias, la conciencia fonológica y el conocimiento de las letras $\left(\mathrm{R}_{\mathrm{CL}-\mathrm{VDL}}^{2}=.76 ; \mathrm{R}^{2}{ }_{\mathrm{VDL}^{-}}{ }_{\mathrm{CL}}=.80\right)$ soportaron el valor predictivo de la velocidad de denominación de letras para la eficiencia lectora. La eficiencia lectora y la velocidad de denominación de letras se relacionaron bidireccionalmente $\left(\mathrm{R}_{\mathrm{VDL}-\mathrm{EL}}^{2}=.34 ; \mathrm{R}_{\mathrm{EL}-\mathrm{VDL}}^{2}=.34\right)$. Por otro lado, la eficiencia lectora y el conocimiento de las letras (que estaba explicado previamente por la conciencia fonológica $\left(\mathrm{R}_{\mathrm{CF}-\mathrm{CL}}^{2}=.78\right)$ establecieron una relación bidireccional $\left(\mathrm{R}_{\mathrm{CL}-\mathrm{EL}}^{2}=.23 ; \mathrm{R}_{\text {EL-CL }}^{2}=.23\right)$. A su vez, la eficiencia lectora era precursor único de las destrezas de la comprensión lectora $\left(\mathrm{R}_{\mathrm{EL}-\mathrm{CLT}}^{2}=.63\right)$. Por último, la comprensión lectora era predicha por las habilidades del lenguaje oral $\left(\mathrm{R}_{\text {LO-CLT }}^{2}=.17\right)$ al final de la secuencia de predicción en el modelo ALICIA. En consecuencia, las habilidades del lenguaje oral adquirieron doble relevancia, tanto en la base del aprendizaje lector como en el soporte de la totalidad del modelo.

En resumen, el análisis de resultados mediante el modelo de ecuaciones estructurales permitió incluir en el modelo de predicción todas las habilidades cognitivas con valor predictivo para el rendimiento lector inicial. Este modelo fue más allá del análisis de regresión lineal, detectando mediaciones entre las variables predictoras y el rendimiento lector. Por esta razón, el modelo ALICIA, que cumplió con los supuestos de bondad de ajuste, incorporó más precursores cognitivos de los que pudieron detectarse mediante 


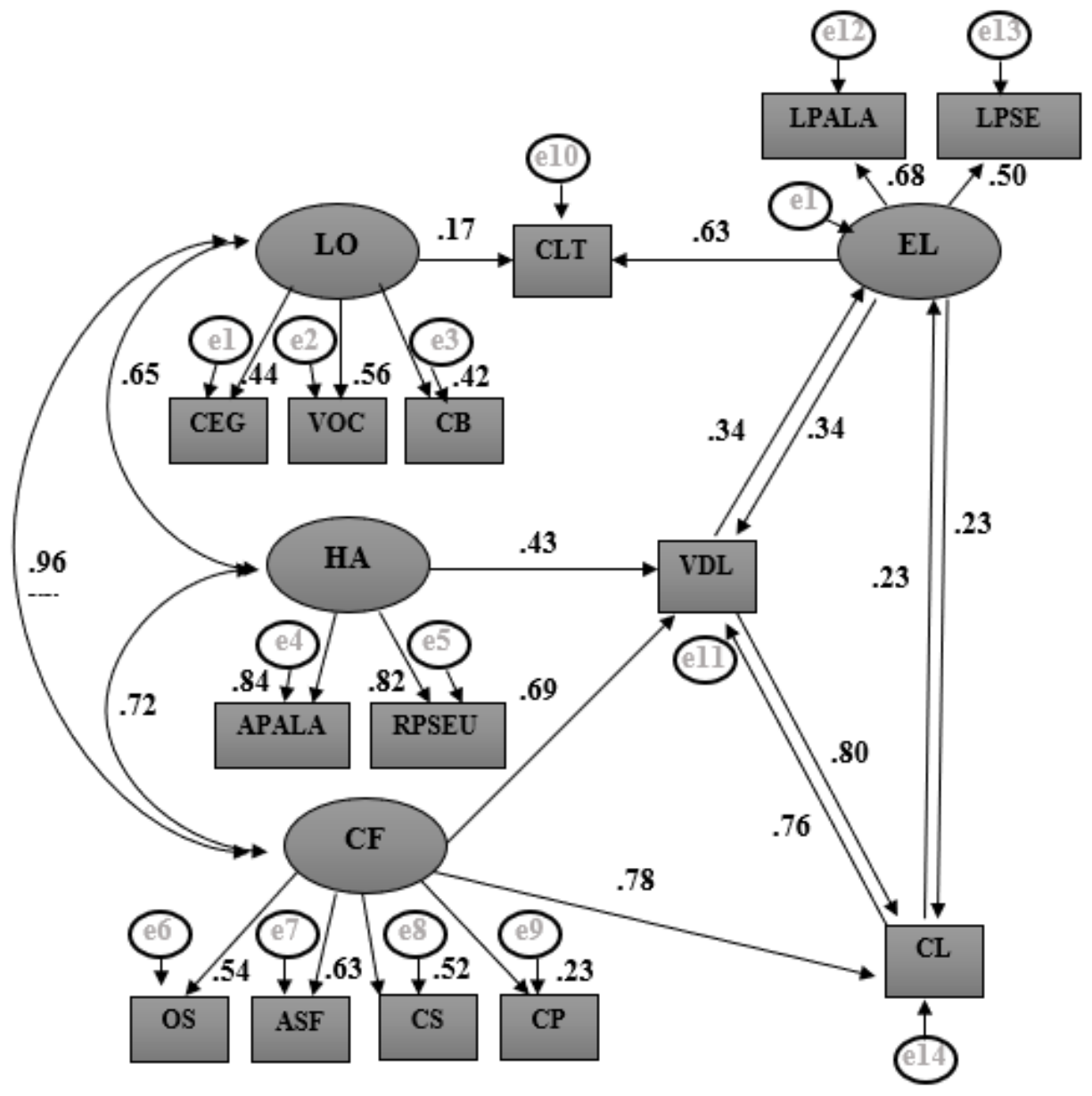

Figura 1. Modelo de ecuaciones estructurales: Modelo ALICIA. LO: Habilidades del lenguaje oral; CEG: Conocimiento de estructuras gramaticales; VOC: Vocabulario; CB: Conceptos básicos; HA: Habilidades articulatorias; APALA: Articulación de palabras; RPSEU: Repetición de pseudopalabras; CF: Conciencia fonológica; OS: Omisión de sílabas; ASF: Aislar sílabas y fonemas; CS: Contar sílabas; CP: Contar palabras; CLT: Comprensión lectora de textos; VDL: Velocidad de denominación de letras; EL: Eficiencia lectora; LPALA: Lectura de palabras; LPSEU: Lectura de pseudopalabras; CL: Conocimiento de letras.

análisis de regresión. Este modelo manifiesta que las contribuciones de las habilidades cognitivas al rendimiento lector fueron más complejas que simples o lineales. El modelo ALICIA subrayó el valor predictivo de las habilidades del lenguaje oral como base del aprendizaje lector, de la velocidad de denominación (en especial, de la velocidad de denominación de letras), del conocimiento de las letras (que aditivamente representa 
la orientación visoespacial de los signos ortográficos), de las habilidades articulatorias y de la conciencia fonológica. Asimismo, el modelo delimitó las relaciones entre las habilidades cognitivas y el rendimiento lector inicial.

\section{Discusión y conclusiones}

Este estudio logró dar respuesta a los tres objetivos planteados. En primer lugar, delimitó un modelo de predicción de la lectura inicial que incluyó como precursores tempranos de la lectura a las habilidades del lenguaje oral, las habilidades articulatorias, la conciencia fonológica, la velocidad de denominación y el conocimiento de las letras. El modelo ALICIA resaltó el valor predictivo de las habilidades del lenguaje oral como base y sustento de la predicción y subrayó para la eficiencia lectora tanto los efectos directos (velocidad de denominación y conocimiento de las letras) como indirectos (conciencia fonológica).

\section{Rol predictivo de las habilidades del lenguaje oral: el inicio del aprendizaje lector}

El modelo de predicción confirmó las conclusiones de los estudios que encontraron el sustento del proceso de adquisición de la lectura en las habilidades del lenguaje oral (Kendeou et al., 2009; Silvén, et al., 2007; Storch \& Whitehurst, 2002). Asimismo, los resultados confirmaron los hallazgos de Catts et al. (2015) y Whitehurst (1996) que mostraron una relación entre el nivel de vocabulario a edades tempranas y el rendimiento lector posterior (Whitehurst \& Lonigan, 1998). El modelo ALICIA subrayó el valor predictivo del vocabulario para la comprensión lectora, en relación directa, y de la eficiencia lectora, en relación indirecta. Este hallazgo apoyó las conclusiones del estudio de Kendeou et al. (2009) que aludieron al valor predictivo del vocabulario en la varianza compartida de las habilidades de descodificación. No obstante, el vocabulario explicó la varianza compartida de la eficiencia lectora en este estudio.

Las habilidades del lenguaje oral favorecieron el desarrollo de otras habilidades cognitivas relacionadas con el código entre los 4 y los 6 años. Las habilidades del lenguaje oral correlacionaron con la conciencia fonológica $(r=.96)$. En este sentido, las conclusiones se asemejaron a aquellas encontradas en las investigaciones de Evans et al. (2006) y Catts et al. (2015) en el mismo intervalo de edad. No obstante, el constructo de habilidades del lenguaje oral en este estudio fue más amplio que el que fue definido por los autores anotados con anterioridad. Esto justificaría que la correlación encontrada en este trabajo fuese mayor a la encontrada en sus resultados.

\section{Las habilidades relacionadas con el código: los mejores precursores cognitivos tempranos de las primeras experiencias lectoras instruccionales en español}

Los resultados mostraron cómo la velocidad de denominación de letras fue el mejor precursor temprano de la lectura inicial en español. Sin embargo, la varianza de la eficiencia lectora explicada fue moderada (R2 =.34). En este sentido, la investigación coincide con las conclusiones de González-Valenzuela et al. (2016) que subrayaron el 
valor predictivo de la velocidad de denominación alfanumérica para la precisión de lectura para palabras y pseudopalabras, y de Schatschneider et al. (2004) que destacaron una contribución única de la velocidad de denominación de letras a los 5 años para la eficiencia lectora de $1^{\circ}(6-7$ años $)(R 2=.43)$. La velocidad de denominación de letras obtuvo mayor valor predictivo que el conocimiento de las letras. De acuerdo con los planteamientos de Neuhaus et al. (2001), se dedujo que las diferencias individuales en el conocimiento de las letras entre los 4 y los 6 años se igualaron con el tiempo. Esto justificó que, por un lado, el conocimiento de las letras entre los 4 y los 6 años hubiera explicado una menor varianza de la eficiencia lectora en $1^{\underline{0}}$ y $2^{\mathrm{o}}$ ( 6 a 8 años) que la hallada para la exactitud lectora (de 5 a 7 años) en el trabajo de Muter et al. (2004) $\left(\mathrm{R}^{2}=.63\right)$; por otro, que hubiese obtenido un valor predictivo similar al alcanzado en la investigación de Torppa et al. (2010) en $2^{\mathrm{o}}$ (8-9 años) $\left(\mathrm{R}^{2}=.34\right)$.

El valor predictivo de la velocidad de denominación de letras residió en sus efectos directos para la eficiencia lectora. Sin embargo, la conciencia fonológica actuó como un precursor indirecto, puesto que esta predijo la velocidad de denominación de letras y el conocimiento de las letras. En este sentido, el modelo no pudo corroborar los hallazgos de los estudios que consideraron a la conciencia fonológica como el mejor precursor de la lectura inicial (Evans et al., 2006; Silvén et al., 2007). Este hallazgo pudo estar motivado por el grado de transparencia lingüística del español. De hecho, esta conclusión podría apoyar que el estudio de Georgiou et al. (2008) hubiese encontrado diferencias en el valor predictivo de la conciencia fonológica y de la velocidad de denominación entre las lenguas inglesa y griega.

La predicción del rendimiento lector derivó del triángulo de relaciones establecidas entre la conciencia fonológica, la velocidad de denominación de letras y el conocimiento de las letras. Esto podría explicar por qué algunos trabajos que combinaron dos de estas habilidades cognitivas a edades tempranas mejoraron el valor predictivo de la lectura en $1^{\circ}$ y $2^{\circ}$ (6-8 años) (Kendeou et al. 2009; Storch \& Whitehurst, 2002). Asimismo, esto justificaría que las tres habilidades cognitivas en su conjunto actuaran como el mejor precursor temprano de la lectura inicial y, en concreto, obtuvieran el mayor valor predictivo para la eficiencia lectora en $1^{\underline{0}}$ (6-7 años) y $2^{\circ}$ en la investigación de Schatschneider et al. (2004).

\section{Modelo de aprendizaje de la lectura inicial - Modelo ALICIA}

El modelo ALICIA ha destacado el rol predictivo de las habilidades del lenguaje oral y de las habilidades relacionadas con el código como el precursor fundamental de las primeras etapas instruccionales del aprendizaje lector. Las habilidades del lenguaje oral, las habilidades articulatorias y la conciencia fonológica correlacionaron y asentaron las bases del proceso lector. Las habilidades articulatorias y la conciencia fonológica explicaron la velocidad de denominación y el conocimiento de las letras, y estas (velocidad de denominación y conocimiento de las letras), a su vez, determinaron la eficiencia lectora. Finalmente, la secuencia predictiva del aprendizaje lector inicial concluyó cuando la eficiencia lectora predijo la comprensión lectora, que fue explicada también por las habilidades del lenguaje oral (figura 2). 


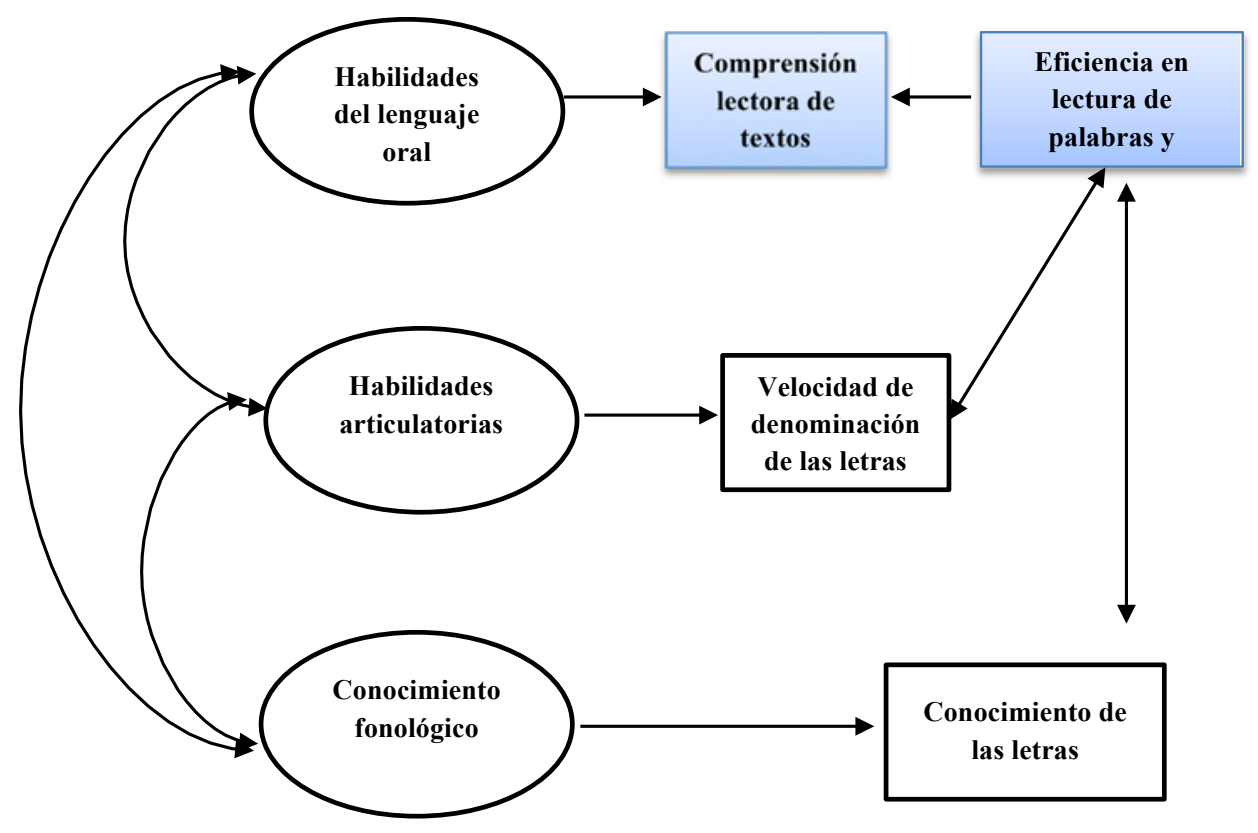

Figura 2. Modelo de aprendizaje de la lectura inicial (en niños españoles de 6 a 8 años).

\section{Limitaciones e implicaciones}

El modelo ALICIA analizó los precursores tempranos de la lectura en las primeras etapas instruccionales del aprendizaje lector en español. El proceso continuo del aprendizaje conllevó la necesidad de acotar en etapas del desarrollo, en este caso, entre los 4 y 8 años. El modelo alcanzado determinó los precursores de la lectura en una etapa delimitada con niños de una idiosincrasia y lengua determinada. La complejidad asumida en el estudio para la delimitación de los precursores cognitivos conllevó el descarte del análisis de los precursores sociales que también fueron importantes (Whitehurst \& Lonigan, 1998). El modelo ALICIA podría presentar variaciones en su valor predictivo al asociarse con otras circunstancias que serán objeto de estudio en futuras investigaciones, tales como las dificultades de aprendizaje o las condiciones sociodemográficas.

\section{Referencias}

Adlof, S. M., Catts, H. W., \& Lee, J. (2010). Kindergarten predictors of second versus eighth grade reading comprehension impairments. Journal of Learning Disabilities, 43(4), 332-345. doi: https://doi.org/10.1177/0022219410369067

Cuetos, F., Rodríguez, B., Ruano, E., \& Arribas, D. (2007). PROLEC-R. Batería de evaluación de los procesos lectores. Revisada. Madrid: TEA. 
Defior, S., Fonseca, L., Gottheil, B., Aldrey, A., Jiménez-Fernández, M. P., Rosa, G., \& Serrano, F. D. (2006). Test de Lectura y Escritura en Español. Buenos Aires, Argentiva: Paidós.

Dickinson, D. K., McCabe, A., Anastasopoulos, L., Peisner-Feinberg, E. S., \& Poe, M. D. (2003). The comprehensive language approach to early literacy: The interrelationships among vocabulary, phonological sensitivity, and print knowledge among preschool-aged children. Journal of Educational Psychology, 95(3), 465-481. doi: https:// doi.org/10.1037/0022-0663.95.3.465

Catts, H. W., Herrera, S., Nielsen, D. C., \& Bridges, M. S. (2015). Early prediction of reading comprehension within the simple view framework. Reading and Writing, 28(9), 1407-1425. doi: https://doi.org/10.1007/s11145-015-9576-x

Evans, M. A., Bell, M., Shaw, D., Moretti, S., \& Page, J. (2006). Letter names, letter sounds and phonological awareness: An examination of kindergarten children across letters and of letters across children. Reading and Writing, 19(9), 959-989. doi: https://doi. org/10.1007/s11145-006-9026-x

Ferroni, M., Diuk, B., \& Mena, M. (2016). Desarrollo de la lectura y la escritura de palabras con ortografía compleja: sus predictores. Avances en Psicología Latinoamericana, 34(2), 253-271. doi:10.12804/apl34.2.2016.04

Georgiou, G. K., Parrila, R., \& Papadopoulos, T. C. (2008). Predictors of word decoding and reading fluency across languages varying in orthographic consistency. Journal of Educational Psychology, 100(3), 566-580. doi: https://doi.org/10.1037/0022-0663.100.3.566

González-Valenzuela, M. J., Díaz-Giráldez, F., \& López-Montiel, M. D. (2016). Cognitive predictors of word and pseudoword reading in Spanish first-grade children. Frontiers in psychology, 7(774),1-12. doi: https://doi.org/10.3389/fpsyg.2016.00774

Kirby, J. R., Desrochers, A., Roth, L., \& Lai, S. S. (2008). Longitudinal predictors of word reading development. Canadian Psychology/Psychologie canadienne, 49(2), 103-110. doi: https://doi.org/10.1037/0708-5591.49.2.103

Frost, J., Madsbjerg, S., Niedersoe, J., Olofsson, A., \& Sorensen, P. M. (2005). Semantic and phonological skills in predicting reading development: From 3-16 years of age. Dyslexia, 11(2), 79-92. doi: https://doi.org/10.1002/dys.292

Kendeou, P., van den Broek, P., White, M. J., \& Lynch, J. S. (2009). Predicting reading comprehension in early elementary school: The independent contributions of oral language and decoding skills. Journal of Educational Psychology, 101(4), 765-778. doi: https://doi.org/10.1037/a0015956

Lonigan, C. J. (2006). Development, assessment, and promotion of preliteracy skills. Early Education and Development, 17(1), 91-114. doi: https://doi.org/10.1207/ s15566935eed1701_5

Lonigan, C. J., Schatschneider, C., Westberg, L., \& The National Early Literacy Panel. (2008). Identification of children's skills and abilities linked to later outcomes in reading, writing, and spelling (Developing early literacy: A scientific synthesis of early literacy development and implications for intervention). Jessup, MD: National Institute for Literacy \& The Partnership

Manis, F., Doi, L., \& Bhadha, B. (2000). Naming speed, phonological awareness, and ortographic knowledge in second graders. Journal of Learning Disabilities, 33(4), 325333, 374. doi: https://doi.org/10.1177/002221940003300405 
Mol, S., Bus, A., \& De Jong, M. (2008). Added Value of Dialogic Parent-Child Book Readings: A Meta-Analysis. Early Education \& Development, 19(1), 7-26. doi: https:// doi.org/0.1080/10409280701838603

Muter, V., Hulme, C., Snowling, M.J., \& Stevenson, J. (2004). Phonemes, rhymes, vocabulary and grammatical skills as foundations of early reading development: Evidence from a longitudinal study. Developmental Psychology, 40(5), 665-681. doi: https://doi. org/10.1037/0012-1649.40.5.665

Neuhaus, G., Foorman, B. R., Francis, D. J., \& Carlson, C. D. (2001). Measures of information processing in rapid automatized naming (RAN) and their relation to reading. Journal of experimental child psychology, 78(4), 359-373. doi: https://doi.org/10.1006/ jecp.2000.2576

Oakhill, J. V., \& Cain, K. (2012). The precursors of reading ability in young readers: Evidence from a four-year longitudinal study. Scientific Studies of Reading, 16(2), 91-121. doi:10.1080/10888438.2010.529219

Paris, S. G. (2005). Reinterpreting the development of reading skills. Reading research quarterly, 40(2), 184-202. doi: https://doi.org/10.1598/RRQ.40.2.3

Schatschneider, C., Fletcher, J., Francis, D., Carlson, C., \& Foorman, B. (2004). Kindergarten prediction of reading skills: A longitudinal comparative analysis. Journal of Educational Psychology, 96(2), 265-282.

Sellés, P., Martínez, T., \& Vidal-Abarca. E. (2010). Batería de Inicio a la lectura: Diseño y características psicométricas. Bordón, 62(2), 137-160.

Silvén, M., Poskiparta, E., Niemi, P., \& Voeten, M. (2007). Precursors of reading skill from infancy to first grade in finnish: Continuity and change in a highly inflected language. Journal of Educational Psychology, 99(3), 516-531. doi: https://doi.org/ doi:10.1037/0022-0663.99.3.516

Storch, S. A., \& Whitehurst, G. J. (2002). Oral language and code-related precursors to reading: Evidence from a longitudinal structural model. Developmental Psychology, 38(6), 934-947.

Suárez-Coalla, P., García-de-Castro, M., \& Cuetos, F. (2013). Variables predictoras de la lectura y la escritura en castellano. Infancia y aprendizaje, 36(1), 77-89. doi: https://doi. org/doi.org/10.1174/021037013804826537

Torppa, M., Lyytinen, P., Erskine, J., Eklund, K., \& Lyytinen, H. (2010). Language development, literacy skills, and predictive connections to reading in Finnish children with and without familial risk for dyslexia. Journal of Learning Disabilities, 43(4), 308-321.

Whitehurst, G. J., \& Lonigan, C. J. (1998). Child development and emergent literacy. Child Development, 69(3), 848-72.

Wolf, M., \& Denckla, M. (2003). Rapid Automatized Naming Tests. Greenville, SC: Super Duper.

Fecha de recepción: 29 de noviembre de 2017.

Fecha de revisión: 4 de diciembre de 2017.

Fecha de aceptación: 15 de abril de 2019. 
